Reprod. Nutr. Dévelop., 1988, 28 (6 B), 1791-1796

\title{
Recherche de facteurs spécifiques dans les milieux de culture des embryons
}

\author{
J. TESTART $\left({ }^{1}\right)$, Clotilde DUOUESNNE $\left({ }^{1}\right)$, Christine WALLON $\left({ }^{2}\right)$, P. GALA- \\ NAUD $\left({ }^{2}\right)$
}

( $\left.{ }^{1}\right)$ INSERMU. 187 et $\left({ }^{2}\right)$ INSERMU. 131, Hôpital Antoine Béclère. 157, rue de la Porte-de-Trivaux, 92140 Clamart, France.

Summary. Search for specific factors in the culture media of human embryos.

Mitogenic activity of culture media having contained either unfertilized or cleaved embryos have been studied. In these media human eggs have been cultured for one day, beginning 20 hours following in vitro insemination.

B-lymphocyte proliferation was estimated by thymidine incorporation following 3 day culture in the presence of eggs or embryo culture media. No reproducible effect on $B$ lymphocyte proliferation was observed. Therefore the viability of embryos could not be ascertained by the mitogenic activity of their culture media.

L'estimation de l'aptitude au développement de l'œuf humain produit par fécondation in vitro (FIV) serait de grande utilité dans les programmes cliniques de FIVETE. Elle permettrait de déterminer, parmi les embryons obtenus, ceux qui doivent être transplantés in utero, soit immédiatement soit après cryopréservation. Ce diagnostic permettrait aussi d'évaluer, en éliminant le paramètre utérin, la qualité des gamètes mis en présence et l'efficacité des méthodes cliniques et biologiques utilisées. Seuls des critères indirects de la vitalité embryonnaire sont éthiquement acceptables, l'analyse destructrice des embryons (morphologie, biochimie) étant exclue. Parmi ces critères indirects l'examen microscopique de l'œuf ne donne que des résultats aléatoires. C'est pourquoi on s'oriente vers l'étude du milieu de culture ayant contenu l'embryon afin d'y déceler les modifications éventuelles caractérisant le métabolisme (Leese, 1987). L'étude des milieux peut également permettre de détecter des facteurs spécifiques tel le PAF (O'Neill, 1985).

Nous utilisons pour la FIV un milieu de culture chimiquement défini (B2) (Ménézo, I.N.R.A., Api-System) pour lequel l'addition de sérum sanguin n'est pas nécessaire (Ménézo et al., 1984). Notre acuité pour déterminer les modifications de ce milieu induites par la présence de l'œuf s'en trouve augmentée, mais la disproportion entre le volume de l'embryon (environ $7 \mu \mathrm{l}$ ) et le volume du milieu utilisé $(0,5$ à $1 \mathrm{ml})$ explique la difficulté à réaliser tout dosage biochimique. C'est pourquoi nous avons tenté de mettre en évidence les effets biologiques des milieux de culture en les faisant agir sur des cellules dont on évalue l'activité mitotique. 
Un premier travail semblait montrer une inhibition de la croissance de fibroblastes humains en présence de B2 ayant contenu un œuf humain, cette inhibition étant 7 fois plus importante quand l'œuf était divisé (Gouga, 1987). Le résultat était estimé par comptage cellulaire après trypsination au $4^{\mathrm{e}}$ jour de culture des fibroblastes.

Dans le présent travail nous avons étudié l'effet des milieux B2 sur la prolifération de lignées lymphoblastoïdes $B$ humaines.

\section{Matériel et méthodes.}

Origine des milieux testés. - II s'agit d'échantillons de $1 \mathrm{ml}$ de milieu B2 ayant contenu un œuf humain entre les $20^{\circ}$ et $44^{\mathrm{e}}$ heures après l'insémination in vitro, les œufs ayant été décoronisés avant d'être introduits dans ces milieux. Certains milieux étaient conservés depuis 1985 à $-30^{\circ} \mathrm{C}$ tandis que d'autres, obtenus en 1988 ont été testés immédiatement. On a comparé avec des milieux contrôle (B2, 1988).

Réalisation du test. — Les lymphocytes B de la lignée lymphoblastoïde RAJI ont été cultivés en plaques de culture multipuits contenant 1500 ou 3000 cellules par puits $(100 \mu \mathrm{l})$.

Différents groupes de culture ont été réalisés :

- RPMI seul (contrôle négatif),

- RPMI + 10\% BCGF (B Cell Growth Factor) (contrôle positif),

- différentes dilutions de milieu B2 diluées dans du RPMI,

- enfin certaines cultures étaient réalisées en l'absence de RPMI, le B2 à tester étant dilué dans du B2 vierge.

Après 3 jours de culture on ajoute la thymidine tritiée $(0,5$ microcuries) pendant $6 \mathrm{~h}$. Après récupération des cellules, l'incorporation de thymidine $\left(\mathrm{H} 3^{*}\right)$ est mesurée avec un compteur à scintillation $\beta$ et les résultats sont obtenus en cpm.

Expression des résultats. - Les résultats sont exprimés en pourcentage de variations entre la moyenne des échantillons expérimentaux et un échantillon témoin. Dans les tableaux ci-dessous l'augmentation (+) ou la diminution ( - ) du marquage ont été symbolisées comme suit : $<25 \%: 0 ; 25$ à $49 \%$ : + ou - ; 50 à $100 \%:++$ ou $--; 100 \%:+++$ ou --- .

\section{Résultats.}

1. Influence de la division de l'œut. - Le tableau 1 montre des résultats extrêmement variables de l'effet des milieux B2 (1985) ayant contenu un œuf soit divisé soit non fécondé sur la multiplication des lymphocytes cultivés en milieu RPMI. Selon la dilution $(1 / 8$ à $1 / 32)$ et la série testée on peut observer une diminution ou une augmentation de plus de $50 \%$ aussi bien pour l'œuf divisé que pour l'ovocyte non fécondé. 
TABLEAU 1

Influence du lot de B2 (1985 ou 1988) et de la division de l'œuf ( $\mathrm{D}=$ divisé, ND = non divisé) sur la multiplication des lymphocytes en milieu RPMI.

\begin{tabular}{|c|c|c|c|c|c|c|c|c|c|}
\hline \multirow{2}{*}{\multicolumn{3}{|c|}{$\begin{array}{l}\text { Concentration cellulaire } / 100 \mu \mathrm{l} \\
\text { dilution milieu testé avec RPMI }\end{array}$}} & & \multicolumn{3}{|c|}{1500} & \multicolumn{3}{|c|}{3000} \\
\hline & & & & $1 / 8$ & $1 / 16$ & $1 / 32$ & $1 / 8$ & $1 / 16$ & $1 / 32$ \\
\hline \multirow[t]{4}{*}{ Milieux } & ND & 1985 & $\begin{array}{l}(n=5) \\
(n=5)\end{array}$ & $\begin{array}{c}+++ \\
0\end{array}$ & $\begin{array}{l}+ \\
0\end{array}$ & $\begin{array}{l}0 \\
0\end{array}$ & $\stackrel{+}{+}$ & $\begin{array}{c}0 \\
++\end{array}$ & -- \\
\hline & D & 1985 & $\begin{array}{l}(n=5) \\
(n=5)\end{array}$ & $\begin{array}{c}+ \\
--\end{array}$ & $\begin{array}{l}+ \\
-\end{array}$ & $\begin{array}{l}0 \\
0\end{array}$ & $\begin{array}{c}++ \\
0\end{array}$ & $\begin{array}{l}0 \\
0\end{array}$ & -- \\
\hline & ND & 1988 & $(n=5)$ & 0 & 0 & 0 & 0 & 0 & 0 \\
\hline & D & 1988 & $(\mathrm{n}=5)$ & 0 & 0 & 0 & 0 & 0 & 0 \\
\hline
\end{tabular}

2. Influence du lot de B2 ou de sa conservation. - Aucun effet de différents milieux de culture (1985 ou 1988) n'est perceptible, que ces milieux aient contenu un œuf divisé ou non fécondé (tabl.1). Ce résultat n'est pas la conséquence de la congélation préalable du milieu testé, des résultats comparables étant obtenus quand le milieu vierge (contrôle) est utilisé soit immédiatement soit après congélation (tabl. 2).

TABLEAU 2

Influence de la congélation du B2 contrôle sur la multiplication des lymphocytes en milieu RPMI.

\begin{tabular}{|c|c|c|c|c|c|c|c|}
\hline \multirow{2}{*}{\multicolumn{2}{|c|}{$\begin{array}{l}\text { Concentration cellulaire/100 } \mu \mathrm{l} \\
\text { dilution B2 avec RPMI }\end{array}$}} & \multicolumn{3}{|c|}{1500} & \multicolumn{3}{|c|}{3000} \\
\hline & & $1 / 8$ & $1 / 16$ & $1 / 32$ & $1 / 8$ & $1 / 15$ & $1 / 32$ \\
\hline $\begin{array}{l}\text { Milieux } B 2 \text { contrô/e } \\
\text { non congelé } 1988 \\
\text { congelé } 1988\end{array}$ & $\begin{array}{l}(n=1) \\
(n=1) \\
(n=1)\end{array}$ & $\begin{array}{l}+++ \\
+++ \\
+++\end{array}$ & $\begin{array}{l}+++ \\
+++ \\
+++\end{array}$ & $\begin{array}{l}+++ \\
++ \\
+++\end{array}$ & $\begin{array}{c}+++ \\
0 \\
++\end{array}$ & $\begin{array}{c}+++ \\
0 \\
+++\end{array}$ & $\begin{array}{c}+++ \\
0 \\
++\end{array}$ \\
\hline
\end{tabular}

On remarque une augmentation plus fréquente de la multiplication lymphocytaire avec les milieux contrôles qu'avec les milieux ayant permis la culture d'un cuf (tabl. 2 versus tabl. 1).

3. Influence des conditions de culture des lymphocytes. - Quand les lymphocytes sont cultivés en milieu B2 exempt de RPMI la même hétérogénéité des réponses à l'addition des milieux de culture d'œuf humain est observée entre les séries expérimentales, sans relation avec la fécondation (tabl. 3) quelles que soient les dilutions des milieux testés (1/8 à 1/2 048).

L'addition de BCGF à la culture des lymphocytes double le taux de multiplication dans la plupart des séries (contrôles et expérimentales) sans relation avec le succès de la fécondation (résultats non montrés). 
Influence de la division de l'œuf ( $\mathrm{D}=$ divisé, $\mathrm{ND}=$ non divisé) sur la multiplication de lymphocytes en milieu B2, exempt de RPMI.

\begin{tabular}{|c|c|c|c|c|c|c|c|c|c|}
\hline \multirow{2}{*}{\multicolumn{2}{|c|}{$\begin{array}{l}\text { Concentration cellulaire/100 } \mathrm{\mu l} \\
\text { Dilution milieu testé avec B2 }\end{array}$}} & \multicolumn{4}{|c|}{1500} & \multicolumn{4}{|c|}{3000} \\
\hline & & $1 / 8$ & $1 / 32$ & $1 / 128$ & $1 / 2048$ & $1 / 8$ & $1 / 32$ & $1 / 128$ & $1 / 2048$ \\
\hline Milieux: & $\begin{array}{r}\text { ND } 1985(\mathrm{n}=5) \\
(\mathrm{n}=4) \\
(\mathrm{n}=5) \\
\text { D } 1985(\mathrm{n}=5)\end{array}$ & $\begin{array}{c}--- \\
--- \\
++ \\
0\end{array}$ & $\begin{array}{l}--- \\
--- \\
+ \\
++\end{array}$ & $\begin{array}{c}--- \\
--- \\
++ \\
0\end{array}$ & $\begin{array}{l}0 \\
+\end{array}$ & $\begin{array}{c}+ \\
++ \\
0 \\
0\end{array}$ & $\begin{array}{l}+ \\
+ \\
0 \\
0\end{array}$ & $\begin{array}{l}+ \\
0 \\
- \\
0\end{array}$ & $\begin{array}{l}0 \\
0\end{array}$ \\
\hline
\end{tabular}

\section{Discussion.}

Une forte prolifération des lymphocytes $B$ apparait après addition de milieu B2 contrôle. Par contre aucune réponse caractérisée (activation ou inhibition) n'est observée de façon stable quand on fait agir le milieu de culture d'un œuf divisé ou non fécondé. Quand, à l'intérieur d'une série expérimentale réalisée le même jour, une différence était perceptible elle consistait le plus souvent en une activation supérieure obtenue après addition de milieux ayant contenu des ovocytes non fécondés par rapport aux milieux ayant contenu des œufs divisés. Toutefois ce résultat restait aléatoire et ne permet aucune conclusion. Dans les conditions où les tests ont été réalisés il apparaît donc impossible de discriminer les œufs humains viables des autres par le pouvoir mitogène de leurs milieux de culture. Cet échec n'est ni la conséquence des dilutions testées, ni l'absence d'un facteur activateur des lymphocytes B (BCGF), de nombreuses autres séries expérimentales non analysées ici ayant exploré ces situations sans succès. L'utilisation de deux autres lignées de lymphocytes cibles (P3HR1 et EBV) a conduit à des résultats analogues. Devant l'hétérogénéité des résultats obtenus pour un même lot de milieu B2, il n'est pas possible de conclure à une variabilité du pouvoir mitogène entre les lots fournis.

La présence de facteur(s) immunosuppresseur(s) dans le milieu de culture d'œufs humains a été rapportée (Daya et Clark, 1986) et des résultats préliminaires ont été obtenus dans l'identification de tels facteurs (Daya et Clark, 1988). Cependant les milieux de culture testés par ces auteurs contenaient $15 \%$ de sérum humain et le test était réalisé sur des lymphocytes $T$ stimulés par la concanavalline $\mathrm{A}$.

Comme pour l'analyse biochimique des milieux de culture (Testart et Ménézo, 1985), la principale difficulté à laquelle se heurtent ces tests biologiques semble être l'extrême dilution d'un éventuel "effet-œuf" dans le milieu de culture. La détection d'un tel effet serait améliorée soit en diminuant le volume du milieu d'incubation de l'œuf soit en augmentant la durée d'incubation. Mais ces éventualités sont difficilement compatibles avec la fonction clinique de la FIVETE. 


\section{Références}

DAYA S., CLARK D. A., 1986. Production of immunosuppressor factor(s) by preimplantation human embryos. Am. J. Reprod. Immun. Microbiol., 11, 98-101.

DAYA S., CLARK D. A., 1988. Identification of two species of suppressive factor of differing molecular weight released by in vitro fertilized human oocytes. Fertil. Steril., 49, 360-363.

GOUGA G., 1987. Méthode d'étude de la vitalité de l'œuf obtenu par fécondation in vitro. Mémoire Concours Internat Pharmacie, Labo. Biochimie, Pr R. LELUC, Hôpital Antoine Béclère, $48 \mathrm{p}$.

LEESE H. J., 1987. Analysis of embryo by non-invasive methods. Human Reprod., 2, 37-40.

MENEZO Y., TESTART J., PERRONE D., 1984. Serum is not necessary in human in vitro fertilization, early embryo culture and transfer. Fertil. Steril., 42, 750-755.

O'NEILL C., 1985. Partial characterization of the embryo-derived platelet-activating factor in mice. J. Reprod. Fert., 75, 375-380.

PUISSANT F., VAN RYSSELBERGE M., BARLOW P., DEWEZE J., LEROY F., 1987 . Embryo scoring as a prognostic tool in IVF treatment. Human Reprod., 2, 705-708.

TESTART J., MEENÉZO Y., 1985. Factors influencing embryo development. In $N^{\text {th }}$ World Congr. $I V F$. Melbourne. 